\title{
Regional Cerebral Blood Flow and BOLD Responses in Conscious and Anesthetized Rats Under Basal and Hypercapnic Conditions: Implications for Functional MRI Studies
}

\author{
Kenneth Sicard, Qiang Shen, Mathew E. Brevard, Ross Sullivan, Craig F. Ferris, Jean A. \\ King, and Timothy Q. Duong \\ Center for Comparative Neurolmaging, Department of Psychiatry, University of Massachusetts \\ Medical School, Worcester, Massachusetts, U.S.A.
}

\section{Summary}

\begin{abstract}
Anesthetics, widely used in magnetic resonance imaging (MRI) studies to avoid movement artifacts, could have profound effects on cerebral blood flow (CBF) and cerebrovascular coupling relative to the awake condition. Quantitative $\mathrm{CBF}$ and tissue oxygenation (blood oxygen level-dependent [BOLD]) were measured, using the continuous arterial-spin-labeling technique with echo-planarimaging acquisition, in awake and anesthetized (2\% isoflurane) rats under basal and hypercapnic conditions. All basal blood gases were within physiologic ranges. Blood pressure, respiration, and heart rates were within physiologic ranges in the awake condition but were depressed under anesthesia $(P<0.05)$. Regional CBF was heterogeneous with whole-brain CBF values of $0.86 \pm 0.25$ and $1.27 \pm 0.29 \mathrm{~mL} \cdot \mathrm{g}^{-1} \cdot \mathrm{min}^{-1}$ under awake and anesthetized conditions, respectively. Surprisingly, CBF was markedly higher (20\% to $70 \%$ across different brain conditions) under isofluraneanesthetized condition compared with the awake state $(P<0.01)$. Hypercapnia decreased $\mathrm{pH}$, and increased $\mathrm{P}_{\mathrm{Co}_{2}}$ and $\mathrm{P}_{2}$. During $5 \% \mathrm{CO}_{2}$ challenge, under awake and anesthetized conditions, respectively, $\mathrm{CBF}$ increased $51 \pm 11 \%$ and $25 \pm 4 \%$, and BOLD increased $7.3 \pm 0.7 \%$ and $5.4 \pm 0.4 \%$. During $10 \% \mathrm{CO}_{2}$ challenge, $\mathrm{CBF}$ increased $158 \pm 28 \%$ and $47 \pm 11 \%$, and BOLD increased $12.5 \pm$ $0.9 \%$ and $7.2 \pm 0.5 \%$. Since CBF and BOLD responses were substantially higher under awake condition whereas blood gases were not statistically different, it was concluded that cerebrovascular reactivity was suppressed by anesthetics. This study also shows that perfusion and perfusion-based functional MRI can be performed in awake animals.
\end{abstract}

\section{Keywords}

CBF; Spin; labeling; fMRI; Awake animals; Anesthesia; Carbon dioxide

\begin{abstract}
Most magnetic resonance imaging (MRI) and spectroscopy studies on animal models involve general anesthesia to eliminate movement artifact. The use of anesthetics, however, could have profound influence on cerebral circulation, metabolism, and neural-vascular coupling. Anesthetics in general are known to suppress neural activity (Ueki et al., 1992), basal cerebral blood flow (CBF) (Ueki et al., 1992), hemodynamic coupling, and functional MRI (fMRI) responses (Lahti et al., 1998; Peeters et al., 2001). Previous studies have shown that basal CBF varies dramatically depending on the anesthetics used. For example, $\mathrm{CBF}$ in rats anesthetized with isoflurane is substantially higher than the CBF in rats anesthetized with fentanyl or
\end{abstract}

Address correspondence and reprint requests to Timothy Q. Duong, Center for Comparative NeuroImaging University of Massachusetts Medical School 55 Lake Ave N, Worcester, MA 01655, U.S.A.; timothy.duong@ umassmed.edu.

K. S. and Q. S. contributed equally to this work. 
pentobarbital (Hendrich et al., 2001). Although many studies have compared CBF and metabolism under different anesthetics, little is known regarding how they differ from conscious condition.

Anesthetics also affect the fMRI signal responses. Functional MRI is widely used as a noninvasive tool to image brain functions ranging from perceptions to cognitive processes in both animal models and humans (Bandettini et al., 1992; Kwong et al., 1992; Ogawa et al., 1992). Functional MRI does not measure neural activity directly; rather, it indirectly detects hemodynamic changes associated with increased neural activity. Therefore, detection of the fMRI signal responses relies on intact coupling between hemodynamic and metabolic responses associated with increased neuronal activity. Such coupling could be markedly affected by anesthetics.

Cerebrovascular coupling has been widely studied using hypercapnic challenge in which carbon dioxide is added to the breathing gas (Weiss et al., 1983; Bandettini and Wong, 1997; Kim and Ugurbil, 1997; Davis et al., 1998; Hoge et al., 1999; Posse et al., 2001). Carbon dioxide, a potent vasodilator and a key modulator of stimulus-induced blood flow response, evokes large CBF and blood oxygen level-dependent (BOLD) increases without concomitant increase in metabolic rate. There is strong evidence that cerebrovascular reactivity to carbon dioxide is also perturbed by anesthetics (Strebel et al., 1993; Bonvento et al., 1994), therefore further supporting the notion that the neural-vascular coupling associated with fMRI is affected by anesthesia.

Although most fMRI studies on animal models are done with the animals under general anesthesia, there is increasing interest in fMRI studies of awake and restrained monkeys (Logothetis et al., 1999; Zhang et al., 2000; Dubowitz et al., 2001; Ferris et al., 2001; Vanduffel et al., 2001), rabbits (Wyrwicz et al., 2000), and rats (Lahti et al., 1998). Functional MRI of awake animals offers many distinct advantages. First, the confounding effects of anesthesia can be avoided. Second, general neural activity is not suppressed (Ueki et al., 1992), which leads to increased fMRI signal changes (Lahti et al., 1998; Peeters et al., 2001) and, potentially improved detection. Finally, subcortical and higher-order cognitive functions can be studied in the awake model. Such studies would be very difficult if not impossible using the anesthetized preparations. The disadvantages of performing awake MRI studies are potential motion artifact and stress.

Although animal studies under anesthetized conditions remain valuable and unavoidable under many circumstances, developing an awake animal model in which perfusion and perfusionbased fMRI can be studied under the fully conscious condition could have many important applications. As a first step to evaluate such an awake rat model, quantitative perfusion was measured using the continuous arterial spin-labeling technique with echo-planar-imaging acquisition to evaluate $\mathrm{CBF}$ in awake and anesthetized rats under basal and hypercapnic challenge. The main goals of this study were (1) to determine whether CBF can be measured under awake and restrained conditions using MRI, (2) to identify major regions of the brain that have substantial perfusion differences between awake and anesthetized conditions, and (3) to evaluate the CBF and BOLD responses to hypercapnia under awake and anesthetized conditions. To the best of our knowledge, this is the first noninvasive measurement of quantitative perfusion and perfusion-based fMRI in fully conscious animals using magnetic resonance imaging. 


\section{Methods}

\section{Animal preparation}

All animal experiments were approved and monitored by the University of Massachusetts Medical Center, Department of Animal Medicine. These procedures were in accordance with guidelines published by the National Institutes of Health. Two groups of Sprague-Dawley rats ( 250 to $300 \mathrm{~g}$ ) were imaged and each rat was studied once. In group $1(\mathrm{n}=8)$, quantitative basal CBF was measured under awake and anesthetized conditions in the same animals. In group $2(n=7), C B F$ and BOLD changes were simultaneously measured in association with hypercapnic $\left(\mathrm{CO}_{2}\right)$ challenges under awake and anesthetized conditions in the same animals. The femoral artery was catheterized for monitoring mean arterial blood pressure, heart rate, and for sampling blood gases. Animals were secured in a rat restrainer with custom-designed ear-, nose-, tooth-, and shoulder-bars, a body-restraining tube, and built-in radiofrequency electronics for imaging, similar to the restrainer reported previously (Lahti et al., 1998). All animals respired without mechanical ventilation. Air or premixed $\mathrm{CO}_{2}$ gas was delivered via a tube to the animal's chamber at a flow rate of $2 \mathrm{~L} / \mathrm{min}$. For the anesthetized condition, $2 \%$ isoflurane was used. The rectal temperature was monitored and maintained at $37 \pm 1{ }^{\circ} \mathrm{C}$ throughout the study via a feedback-regulated, circulating-water pad.

Physiologic measurements-Mean arterial blood pressure, heart rate, and respiration rate were continuously monitored and recorded. These parameters were quantified under basal and hypercapnic conditions in awake and anesthetized rats during imaging. Blood gases were sampled immediately at the end of MRI measurement of each $\mathrm{CO}_{2}$ challenge while the animals were in the magnet, to avoid the effect of withdrawing blood on the MR signals.

Hypercapnic challenges (group 2)-For each animal, two (5 and 10\%) carbon dioxide concentrations with $21 \% \mathrm{O}_{2}$ and balance $\mathrm{N}_{2}$ were studied under anesthetized and awake conditions. In each MRI experiment ( $\approx 200$ seconds), the animal was exposed to air (baseline) for 40 seconds, followed by $5 \%$ or $10 \% \mathrm{CO}_{2}$ gas for 160 seconds. Typically, at least 15 minutes elapsed between each $\mathrm{CO}_{2}$ challenge and between awake and anesthetized conditions.

Magnetic resonance experiments-All MR experiments were performed on a 4.7-T/40$\mathrm{cm}$ horizontal magnet (Oxford Instrument, Oxford, U.K.) equipped with a Biospec Bruker console (Bruker, Billerica, MA, U.S.A.) and a 20-G/cm magnetic field gradient insert (ID = $12 \mathrm{~cm}$ ) capable of $120-\mu$ s rise time (Bruker). An actively decoupled surface coil (2.3-cm inner diameter) was used for brain imaging and a neck coil (Silva et al., 1999; Duong et al., 2000b) for perfusion labeling. Coil-to-coil electromagnetic interaction was actively decoupled. Anatomic images were acquired using the fast spin-echo (rapid acquisition with relaxation enhancement) pulse sequence with repetition time $(\mathrm{TR})=2$ seconds $\left(90^{\circ}\right.$ flip angle $), 8$ echo trains, effective echo time $(\mathrm{TE})=65$ milliseconds, matrix $=256 \times 256$, field of view $=2.5 \times$ $2.5 \mathrm{~cm}^{2}$, and six $1.5-\mathrm{mm}$ slices. Four transients were acquired for signal averaging.

Cerebral blood flow measurements were made using the continuous arterial spin-labeling technique (Silva et al., 1999; Duong et al., 2000b) with single-shot, gradient-echo, echo-planarimaging acquisition. Paired images were acquired alternately: one with arterial spin labeling and the other without spin labeling (control). The MR parameters were: data matrix $=64 \times 64$, field of view $=2.5 \times 2.5 \mathrm{~cm}^{2}$, and six 1.5 -mm slices, $\mathrm{TE}=15$ milliseconds, and $\mathrm{TR}=2$ seconds $\left(90^{\circ}\right.$ flip angle). Continuous arterial spin labeling used a 1.78-second square radiofrequency pulse to the labeling coil in the presence of $1.0 \mathrm{G} / \mathrm{cm}$ gradient along the flow direction such that the condition of adiabatic inversion was satisfied (Detre et al., 1992). The sign of the frequency offset was switched for control (nonlabeled) images. For each set of CBF 
measurement, 51 pairs of images $(\approx 200$ seconds) were acquired and the first two pairs were discarded and excluded from analysis.

Blood oxygenation level-dependent images were taken from the nonlabeled (control) images of the CBF measurements, and no additional data acquisition was needed. Note that the optimal gradient-echo BOLD contrast is obtained with echo time set at tissue $\mathrm{T}_{2}{ }^{*}$, which is $\approx 20$ milliseconds at 4.7 T. In this study, the echo time was 15 milliseconds, which was chosen to compromise between BOLD contrast and CBF signal-to-noise ratio. Thus, larger BOLD percent changes would be expected if the optimal echo time was used. Nevertheless, the overall conclusions in this study were not altered.

For group 1, each CBF measurement set (51 pairs) was typically repeated three times and acquired alternately between awake and anesthetized conditions. For group 2, each CBF measurement set (51 pairs) was typically repeated twice for each gas condition and acquired alternately between awake and anesthetized conditions.

Data analysis-Image analysis used codes written in Matlab (MathWorks Inc., Natick, MA, U.S.A.) and the STIMULATE software (Strupp, 1996). All statistical tests used the Student's paired $t$-test. All reported values and error bars on plots were in mean \pm SD.

For group 1, $\mathrm{CBF}$ image $\left(\mathrm{S}_{\mathrm{CBF}}\right)$ with intensity in milliliters per gram tissue per minute was calculated pixel-by-pixel using (Silva et al., 1997b)

$$
S_{C B F}=\frac{\lambda}{T_{1}} \frac{S_{C}-S_{L}}{S_{L}+(2 \alpha-1) S_{C}}
$$

where $S_{C}$ and $S_{L}$ are signal intensities of the control and labeled images, respectively. $\lambda$ is the water brain-blood partition coefficient, $T_{1}$ is that of tissue, and $\alpha$ is the arterial spin-labeling efficiency (Silva et al., 1997b). $\lambda$ was taken to be 0.9 (Herscovitch and Raichle, 1985). $T_{1}$ and $\alpha$ were measured and they were 1.5 seconds and 0.75 , respectively.

Multiple CBF measurements from each animal were averaged to obtain a single multislice set of awake CBF images and a single multislice set of anesthetized CBF images. Alignment of CBF images in a study was checked by visually evaluating the time-series "movies" created by appending images acquired at different time points. Regions of interests (ROIs) were analyzed in both hemispheres and were carefully drawn with references to both anatomy and echo-planar images. These ROIs were cerebral cortex, corpus callosum, hippocampus, thalamus, and caudoputamen. The same ROIs were used for the awake and anesthetized conditions in the same animal. Subtraction maps between awake and anesthetized rats were also calculated and color-coded.

For group 2, hypercapnia-induced CBF and BOLD changes were analyzed. Conservative ROIs enclosing the entire brain but avoiding the edge of the brain were used. Percent change maps were obtained by using $t$-test analysis with images 2 to 11 as baseline and images 16 to 51 as stimulation. Images 12 to 15 were not included in the $t$-test because $\mathrm{CO}_{2}$ and fMRI signals were dynamically changing and not at steady state.

Functional contrast-to-noise ratios were calculated pixel-by-pixel as the hypercapnia-evoked percent changes divided by the standard deviations of the baseline images before $\mathrm{CO}_{2}$ challenge. Rise times were not calculated because of the relatively long TR used in this study. 


\section{Results}

\section{Physiologic measurement under awake and anesthetized conditions}

The number of struggling movements as well as the number of vocalizations markedly decreased after the first hour of being awake in the restrainer and remained low during the entire MR study, suggesting that the animals had acclimated to the restrainer to some extent. Therefore, all MR and physiologic measurements were made with animals being awake for at least $1 \mathrm{~h}$ in the restrainer.

Physiologic measurements under basal and hypercapnic conditions are summarized in Table 1. Comparisons of these physiologic parameters between awake and anesthetized conditions were made in the same animals. Blood $\mathrm{pH}, \mathrm{P}_{\mathrm{CO}_{2}}, \mathrm{P}_{2}$ and oxygenation saturation under both awake and anesthetized conditions were within normal physiologic ranges (Flecknell and Waynsorth, 1992;Sharp and LaRegina, 1998) and these parameters were not statistically different between awake and anesthetized rats $(P>0.05)$. The respiration rate, heart rate, and mean arterial blood pressure under the awake condition were consistent with established data in rats (Flecknell and Waynsorth, 1992;Sharp and LaRegina, 1998), where the respiration rate was reported to range from 70 to 115 breaths per minute, heart rate 300 to 450 beats per minute, systolic blood pressure 116 to $180 \mathrm{~mm} \mathrm{Hg}$ and diastolic blood pressure 60 to $145 \mathrm{~mm} \mathrm{Hg}$. The respiration rate, heart rate, and mean arterial blood pressure under isoflurane anesthesia, although slightly depressed $(P<0.05)$, were within normal physiologic ranges.

Under hypercapnic condition (also shown in Table 1), there were no statistical differences in blood-gas values between awake and anesthetized conditions for each $\mathrm{CO}_{2}$ gas conditions; for example, under $5 \% \mathrm{CO}_{2}$ challenge, blood-gas values were not statistically different between awake and anesthetized conditions. During hypercapnia, $\mathrm{pH}$ decreased, and $\mathrm{P}_{\mathrm{co} 2}$ and $\mathrm{P}_{2}$ increased; however, there were no statistical differences in heart rate, mean arterial blood pressure, and respiration rate relative to the basal condition. A general increase in respiration volume was observed during hypercapnia (data not shown).

\section{Basal cerebral blood flow differences between awake and anesthetized rats}

Representative anatomic images obtained from an awake rat are shown in Fig. 1A. There were no obvious movement artifacts in the anatomic images. Superimposed on these anatomic images are ROIs of five major brain structures used in our quantitative CBF analysis, namely, the cerebral cortex, caudoputamen, thalamus, corpus callosum, and hippocampus.

Representative single-shot, echo-planar images obtained from an awake rat are shown in Fig. 1B. No gross misalignment was observed in the time-series "movies" generated from multiple repeated measurements.

Representative quantitative CBF images from a rat under awake and anesthetized conditions are shown in Fig. 1C and 1D, respectively. Under both awake and anesthetized conditions, $\mathrm{CBF}$ was heterogeneously distributed, likely attributed to regional differences in metabolic rate (Sokoloff et al., 1977) and/or vascular density (Zeman and Innes, 1963; Miyawaki et al., 1998). Surprisingly, global CBF values were significantly lower in the awake condition relative to the isoflurane-anesthetized condition in the same rats. Cerebral blood flow values in different brain regions ranged from 0.9 to $1.44 \mathrm{~mL} \cdot \mathrm{g}^{-1} \cdot \min ^{-1}$ (whole-brain average of $1.27 \pm 0.29$ $\mathrm{mL} \cdot \mathrm{g}^{-1} \cdot \mathrm{min}^{-1}$ ) under isoflurane-anesthetized condition, and ranged from 0.6 to $1.2 \mathrm{~mL}$. $\mathrm{g}^{-1} \cdot \min ^{-1}$ (whole-brain average of $0.86 \pm 0.25 \mathrm{~mL} \cdot \mathrm{g}^{-1} \cdot \mathrm{min}^{-1}$ ) in the awake state.

Furthermore, the CBF subtraction maps (Fig. 1E) of the anesthetized condition from awake condition shows that there were heterogeneous differences between awake and anesthetized $\mathrm{CBF}$. Regions that were less negative (smaller CBF difference [i.e., blue pixels]) in the CBF 
subtraction maps were indicative of areas with differentially higher basal CBF in the awake condition.

A typical block of CBF measurement over $\approx 3.3$ minutes is shown in Fig. 1F. The CBF-weighted intensities, alternating between nonlabeled and labeled images, are indicative of the presence of labeled blood flow. The CBF contrast, derived from subtracting labeled from nonlabeled image, was $\approx 8 \%$ of the original signal, ranging from $4 \%$ to $10 \%$ for all animals. These data further showed that there were no obvious movement artifacts in individual CBF measurements under the awake condition.

Although there was some partial volume effect due to limited spatial resolution, many major brain structures were clearly identified. Quantitative CBF values for five major brain substructures from all eight rats were quantified and are summarized in Fig. 2. Among these five structures, the largest differences in CBF between awake and anesthetized rats were in the hippocampus and caudoputamen $(\approx 70 \%)$ and the smallest difference in CBF between awake and anesthetized rats was in the cerebral cortex $(\approx 20 \%)$. The corpus callosum and thalamic nuclei displayed intermediate difference of the former and latter.

\section{Hypercapnia-induced cerebral blood flow changes in awake and anesthetized rats}

Cerebral blood flow and BOLD responses to hypercapnia were evaluated under awake and anesthetized conditions in the same animals and are summarized in Fig. 3. Hypercapnia-evoked $\mathrm{CBF}$ and BOLD responses were readily detected without movement artifacts. During 5\% $\mathrm{CO}_{2}$ challenge, under awake and anesthetized conditions, respectively, CBF increased $51 \pm$ $11 \%$ and $25 \pm 4 \%$, and BOLD increased $7.3 \pm 0.7 \%$ and $5.4 \pm 0.4 \%$. During $10 \% \mathrm{CO}_{2}$ challenge, CBF increased $158 \pm 28 \%$ and $47 \pm 11 \%$, and BOLD increased $12.5 \pm 0.9 \%$ and $7.2 \pm 0.5 \%$. Hypercapnia-induced CBF and BOLD changes were markedly higher under awake condition $(P<0.05)$, whereas blood gases were not statistically different, suggesting that cerebrovascular reactivity to $\mathrm{CO}_{2}$ was markedly attenuated by anesthesia. It is noteworthy that CBF and BOLD percent changes during $5 \% \mathrm{CO}_{2}$ challenge under the awake state were comparable to those with the $10 \% \mathrm{CO}_{2}$ challenge under the anesthetized conditions. Hypercapnia-induced CBF changes under the awake condition were 2.0 to 3.3 times greater than that observed in the anesthetized condition, whereas hypercapnia-induced BOLD changes under the awake condition were only 1.3 to 1.7 times larger. The differential responses in CBF and BOLD could be indicative of the underlying metabolic differences between the awake and anesthetized conditions.

Basal MRI signal fluctuation under the awake condition was substantially higher than that under the anesthetized condition. Higher basal signal fluctuations under the awake condition, however, did not necessarily arise from increased movement; they were likely a result of increased basal neural activity and/or increased physiologic "processes" associated with being awake. Functional contrast-to-noise ratios (CNRs) were compared. For the BOLD signals, the CNRs were $20 \pm 5$ for the $10 \% \mathrm{CO}_{2}$ condition and $10 \pm 7$ for the $5 \% \mathrm{CO}_{2}$ conditions under the awake state; $16 \pm 5$ for the $10 \% \mathrm{CO}_{2}$ conditions and $10 \pm 6$ for the $5 \% \mathrm{CO}_{2}$ conditions under the anesthetized condition. For the CBF signals, CNRs were $14 \pm 8$ for the $10 \% \mathrm{CO}_{2}$ condition and $8 \pm 4$ for the $5 \% \mathrm{CO}_{2}$ conditions under the awake state; $6 \pm 3$ for the $10 \% \mathrm{CO}_{2}$ conditions and $4 \pm 2$ for the $5 \% \mathrm{CO}_{2}$ conditions under the anesthetized condition. Contrast-to-noise ratios were statistically different between $5 \%$ and $10 \% \mathrm{CO}_{2}$ challenges $(P<0.05)$ but not statistically different between awake and anesthetized conditions with the same $\mathrm{CO}_{2}$ challenge $(P>0.05)$. Although the BOLD CNRs were higher overall, there were no statistical differences between BOLD and CBF contrasts. We concluded that the CNRs were comparable between awake and anesthetized conditions, at least under these experimental conditions. 


\section{Discussion}

\section{Quantitative perfusion in conscious animals}

Despite the widespread use of anesthetics in experimental animal models and in human studies, the effect of anesthetics on CBF remains poorly understood. Although all anesthetics affect basal CBF, metabolism, neural activity, and neural-vascular coupling, little is known regarding the extent to which CBF is modulated globally and in different regions of the brain under anesthesia relative to the awake condition.

Magnetic resonance imaging offers a noninvasive tool to image quantitative $\mathrm{CBF}$ at reasonably high spatial and temporal resolution at the level of the capillary and tissue (Kwong et al., 1992; Kim, 1995; Wong et al., 1998; Duong et al., 2001c). Although consistent with many established invasive $\mathrm{CBF}$ techniques, the accuracy of the continuous arterial spin-labeling CBF technique could be subjected to errors arising from magnetization transfer, transit time, and water-exchange effect. With the use of the actively decoupled two-coil system for CBF measurements, the magnetization transfer effect on quantitative CBF values was not an issue (Silva et al., 1999; Duong et al., 2000b). Transit time (Calamante et al., 1999; Zhou and van Zijl, 1999) and water-exchange effect (Silva et al., 1997a; Zhou et al., 2001; Parkes and Tofts, 2002), however, could potentially cause errors in the quantitative blood flow values. These errors, nevertheless, are likely to be small (Silva et al., 1997a; Zhou et al., 2001; Parkes and Tofts, 2002) and are not expected to alter the overall conclusions of this study. The continuous arterial spin-labeling technique used herein has also been validated using iodoantipyrine autoradiography (Tsekos et al., 1998).

Most magnetic resonance imaging and spectroscopy studies on animal models involve general anesthesia to avoid movement artifacts. Perfusion imaging is particularly sensitive to movement artifacts because it has low signal-to-noise ratio and it involves paired images acquired at two different time points during which movement could occur. The use of a proper restrainer has provided quantitative perfusion images free of movement artifacts. In addition, data acquisition using the echo-planar-imaging technique was helpful in minimizing the effects of movement artifacts in MR images because this imaging method samples the entire k-space in a single-shot acquisition within $\approx 20$ milliseconds. Therefore, such an approach offers a means to quantitatively evaluate regional CBF in fully conscious rats, allowing direct comparison of quantitative $\mathrm{CBF}$ in different regions of the brain under awake and anesthetized conditions in the same animals.

Restraint could have a dramatic effect on animal physiology. However, blood gases, heart rate, respiration rate, and mean arterial blood pressure under the awake preparations were within normal physiologic ranges, and the number of struggling movements markedly decreased after the first hour of being awake in the restrainer. Taken together, these observations suggest that the animals have substantially acclimated to the restrainer during the imaging session. Detailed investigation of the acclimation and restraint effect is currently under investigation.

Nevertheless, it is important to keep in mind that the regional difference in CBF values could be influenced by restraint stress.

Awake fMRI studies have used the BOLD technique to detect oxygenation changes (Lahti et al., 1998; Logothetis et al., 1999; Wyrwicz et al., 2000; Ferris et al., 2001) and the monocrystalline iron oxide nanoparticles technique to detect blood-volume changes (Dubowitz et al., 2001; Vanduffel et al., 2001) associated with increased neural activity. This study further showed that perfusion and perfusion-based functional imaging could be performed in fully conscious and restrained animals. Although awake perfusion imaging is without movement artifacts and there are indications that rats had acclimated to the restrainer to some extent, the issue of stress nevertheless needs to be fully addressed. 


\section{Effect of anesthesia on cerebral blood flow}

Quantitative perfusion in different regions of the brain could be reliably measured within and across different animals under both anesthetized and conscious conditions. The average perfusion value across the entire brain was $1.27 \mathrm{~mL} \cdot \mathrm{g}^{-1} \cdot \mathrm{min}^{-1}$ under the isofluraneanesthetized condition. These results are consistent with a rat study under $2 \%$ isoflurane (mechanically ventilated) (Duong et al., 2001b) and the Hendrich et al. (2001) study in rats under $1 \%$ isoflurane (mechanically ventilated in the presence of pancuronium paralytics). Cerebral blood flow values in the awake condition were similar to those under chloral hydrate $\left(\approx 1.14 \pm 0.11 \mathrm{~mL} \cdot \mathrm{g}^{-1} \cdot \min ^{-1}, \mathrm{n}=6\right.$, unpublished data). By contrast, $\mathrm{CBF}$ in the rat brain under $\alpha$-chloralose was substantially lower $\left(\approx 0.64 \pm 0.17 \mathrm{~mL} \cdot \mathrm{g}^{-1} \cdot \mathrm{min}^{-1}\right.$ using MRI (Duong et al., 2000b) and $0.72 \pm 0.19 \mathrm{~mL} \cdot \mathrm{g}^{-1} \cdot \mathrm{min}^{-1}$ using iodoantipyrine autoradiography (Tsekos et al., 1998). Cerebral blood flow under pentobarbital is generally even lower (Hendrich et al., 2001). It should be noted that CBF are critically dependent on anesthetic doses. Although there are cross-laboratory differences in animal preparations, blood-gas condition, and anesthetic dose (among others), these comparisons clearly showed that anesthetics have powerful influences on basal CBF.

Literature on CBF comparison between awake and anesthetized conditions is sparse. Cerebral blood flow under awake and restrained conditions in animal models had been measured using autoradiographic techniques (Dahlgren et al., 1981; Young et al., 1991), xenon washout technique (Reinstrup et al., 1995), microsphere technique (Boarini et al., 1984), hydrogen clearance technique (Heiss and Traupe, 1981), and single-photon emission computer-aided tomography (Reinstrup et al., 1995). Most of the aforementioned techniques, however, are highly invasive and yield poor spatial localization, which makes it difficult to quantify and compare $\mathrm{CBF}$ in various brain structures. Furthermore, no systematic comparison of CBF under awake and anesthetized conditions was made using the existing invasive techniques in the same animals and in the same regions of interest.

One important and surprising observation is that $\mathrm{CBF}$ across the entire brain under isofluraneanesthetized condition was consistently higher (20\% to $70 \%$ across different brain regions) than that measured in the awake state. Isoflurane is known to suppress overall electroencephalographic activity (Maekawa et al., 1986) and cerebral metabolism (Sokoloff et al., 1977; Stullken et al., 1977; Wei et al., 1993) relative to the awake condition, and yet surprisingly it yields higher CBF. Higher CBF under isoflurane relative to the awake condition has been previously reported. In a study in which radionuclide-labeled microspheres were used to measure $\mathrm{CBF}$ in pigs, $\mathrm{CBF}$ under $1.45 \%$ isoflurane was reported to be $120 \%$ to $145 \%$ of that under the awake condition (Manohar and Parks, 1984). Similarly, Hoffman et al. (1991) reported comparable $\mathrm{CBF}$ increases in rats under isoflurane anesthesia relative to the awake condition. These observations suggest that the intricate neural-vascular coupling is perturbed by isoflurane (i.e., $\mathrm{CBF}$ is not commensurate with neural activity). The most likely explanation is that isoflurane is a potent cerebro-vasodilator, which leads to a global increase in perfusion. Isoflurane's being a strong cerebro-vasodilator had been demonstrated (Hensen et al., 1988; Matta et al., 1999). In addition to a global CBF difference between awake and anesthetized conditions, there are regional differences in CBF between awake and anesthetized conditions, suggesting that there are intrinsic regional differences in metabolism and/or neural activity between the awake and anesthetized conditions, as expected. Regions that have higher metabolism and neural activity (pixels with smaller negative differences in Fig. 1E) under the awake condition relative to the anesthetized condition were the cortex, the corpus callosum, and the thalamus. Preferential higher basal CBF in these three structures in the awake condition are not unexpected. Cortex generally has higher CBF compared with most other brain structures, probably because of the high basal metabolic activity for processing sensory information. Evidently, there is increased sensory processing in the awake condition. The 
thalamus, whose primary role is to integrate signals from various brain regions and is the gatekeeper of the cortical input and output, is also expected to increase activity under the awake condition. Finally, increased activity associated with being awake also requires increased communication between the two cerebral hemispheres, thus leading to increased CBF in the corpus callosum under awake condition.

\section{Implications in stroke and functional magnetic resonance imaging studies}

Anesthetics also affect stroke outcome (Jacewicz and Pulsinelli, 1986; Molinari, 1986; Warner et al., 1995). Both halothane and sevoflurane reduce ischemic damage relative to that incurred in awake rats (Warner et al., 1993). Similarly, isoflurane has been shown to attenuate delayed development of selective neuronal necrosis in periinfarct areas (Kawaguchi et al., 2000). A recent study showed that concurrent or delayed administration of propofol is neuroprotective relative to the awake preparations (Gelb et al., 2002). Furthermore, although numerous therapeutic drugs showed positive results in experimental stroke, none has proven to be effective clinically, especially in terms of neuroprotection (De-Graba and Pettigrew, 2000; Liebeskind and Kasner, 2001). Although there are many possible reasons why this may be so, one crucial factor is that the models to date have been done in anesthetized animals and, thus, anesthesia could be a confounding factor.

Anesthetics could affect fMRI signals by suppressing neural activity, perturbing the neuralvascular coupling associated with increased neural activity and/or modulating basal blood flow. Numerous studies have shown that anesthetics in general, and isoflurane in particular, decrease glucose and oxygen metabolism (Stullken et al., 1977) and EEG activity (Maekawa et al., 1986). Our results showed that the cerebrovascular reactivity was markedly attenuated by isoflurane relative to the awake condition. This observation is consistent with previous studies in which blood flow on the cortical surface was measured using laser Doppler flowmetry. In those studies, it was reported that the vascular reactivity to carbon dioxide under halothane (Bonvento et al., 1994) and isoflurane (Strebel et al., 1993) was markedly reduced relative to the awake condition. Similarly, in another study in which blood flow in the middle cerebral artery was measured using the transcranial Doppler method, ketamine was found to attenuate cerebrovascular response to carbon dioxide in humans (Nagase et al., 2001).

Anesthetics also have an effect on the fMRI signals per se because basal blood flow modulates the magnitude and the dynamics of the BOLD responses (i.e., time to peak and full-width at half maximum of the fMRI response function). It has been shown that a higher basal CBF (induced by hypercapnia) yields a smaller stimulus-evoked BOLD percent change and a slower, broader hemodynamic response associated with visual stimulation (Kemna and Posse, 2001; Cohen et al., 2002). Therefore, the BOLD and CBF responses are expected to be dependent on the anesthetics used.

Anesthetics also have important implications in using the early negative BOLD (the initial dip) to map brain functions. Although many laboratories have shown the existence of the initial dip in humans (Menon et al., 1995; Hu et al., 1997) and in animal models (Duong et al., 2000a; Kim et al., 2000), and high-resolution mapping of columnar structures has been reported using the initial dip (Duong et al., 2000a; Kim et al., 2000), the initial dip remains controversial (Buxton, 2001). The inconsistency across laboratories regarding the existence of the early negative BOLD could arise from many factors, including nature of the stimuli, stimulation duration, areas of the brain stimulated, animal models, data analysis, and anesthetics. For example, in humans, the early negative BOLD can only be detected using a "model function" (Yacoub et al., 1999), and the early dip is completely averaged out if all pixels that show a positive response are considered (Menon et al., 1995; Hu et al., 1997). In marked contrast, the negative BOLD response can be readily detected with an ROI over the entire visual cortex in an anesthetized cat model (Duong et al., 2000a; Kim et al., 2000). It has been 
further shown that the CBF response to stimuli is markedly delayed and sluggish under isoflurane in the cat model, likely to accentuate the initial dip (Duong et al., 2001a; Kim and Duong, 2002). This comparison suggests that anesthetics could be a major factor, namely, isoflurane anesthesia could cause a delayed CBF increase (i.e., sluggish CBF responses), resulting in the initial dip. Further investigation is warranted.

\section{Conclusions}

Surprisingly, basal CBF under the awake condition was substantially lower than that under isoflurane anesthesia. Regional CBF differences between awake and anesthetized rats were rather heterogeneous. The hypercapnia-evoked CBF and BOLD percent changes were substantially higher in the awake than in the anesthetized condition, suggesting that anesthesia markedly suppressed cerebrovascular reactivity to carbon dioxide and thus neural-vascular coupling. This study showed that quantitative perfusion imaging has the potential to be used to investigate brain functions and cerebral ischemia in fully conscious animals.

\section{Acknowledgments}

This study was supported in part by the Whitaker Foundation.

\section{References}

Bandettini PA, Wong EC. A hypercapnia-based normalization method for improved spatial localization of human brain activation with fMRI. NMR Biomed 1997;10:197-203. [PubMed: 9430348]

Bandettini PA, Wong EC, Hinks RS, Rikofsky RS, Hyde JS. Time course EPI of human brain function during task activation. Magn Reson Med 1992;25:390-397. [PubMed: 1614324]

Boarini DJ, Kassell NF, Coester HC, Butler M, Sokoll MD. Comparison of systemic and cerebrovascular effect of isoflurane and halothane. Neurosurgery 1984;15:400-409. [PubMed: 6483155]

Bonvento G, Charbonne R, Correze JL, Borredon J, Seylaz J, Lacombe P. Is alpha-chloralose plus halothane induction a suitable anesthetic regimen for cerebrovascular research? Brain Res 1994;665:213-221. [PubMed: 7895056]

Buxton RB. The elusive initial dip. Neuroimage 2001;13:953-954. [PubMed: 11352601]

Calamante F, Thomas DL, Pell GS, Weiersma J, Turner R. Measuring cerebral blood flow using magnetic resonance imaging techniques. J Cereb Blood Flow Metab 1999;19:701-735. [PubMed: 10413026]

Cohen ER, Ugurbil K, Kim SG. Effect of basal conditions on the magnitude and dynamics of the blood oxygenation level-dependent fMRI response. J Cereb Blood Flow Metab 2002;22:1042-1053. [PubMed: 12218410]

Dahlgren N, Ingvar M, Yokoyama HJ, Siesjo BK. Effect of indomethacin on local cerebral blood flow in awake, minimally restrained rats. J Cereb Blood Flow Metab 1981;1:233-236. [PubMed: 7328141]

Davis TL, Kwong KK, Weisskoff RM, Rosen BR. Calibrated functional MRI: Mapping the dynamics of oxidative metabolism. Proc Natl Acad Sci U S A 1998;95:1834-1839. [PubMed: 9465103]

DeGraba TJ, Pettigrew LC. Why do neuroprotective drugs work in animals but not humans? Neurol Clin 2000;18:475-493. [PubMed: 10757837]

Detre JA, Leigh JS, Williams DS, Koretsky AP. Perfusion imaging. Magn Reson Med 1992;23:37-45. [PubMed: 1734182]

Dubowitz DJ, Bernheim KA, Chen DY, Bradley JWG, Andersen RA. Enhancing fMRI contrast in awakebehaving primates using intravascular magnetite dextran nanoparticles. Neuroreport 2001;12:23352340. [PubMed: 11496106]

Duong TQ, Kim DS, Ugurbil K, Kim SG. Spatio-temporal dynamics of the BOLD fMRI signals in cat visual cortex: Toward mapping columnar structures using the early negative response. Magn Reson Med 2000a;44:231-242. [PubMed: 10918322]

Duong TQ, Silva AC, Lee SP, Kim SG. Functional MRI of calcium-dependent synaptic activity: cross correlation with CBF and BOLD measurements. Magn Reson Med 2000b;43:338-392. 
Duong TQ, Harel N, Kim SG. Does the early-negative BOLD signal arise from a delayed CBF on set? Proc Int Soc Magn Reson Med 2001a;2:1161.

Duong TQ, Iadacola C, Kim SG. Effect of hyperoxia, hypercapnia and hypoxia on cerebral interstitial oxygen tension and cerebral blood flow in the rat brain: an ${ }^{19} \mathrm{~F} /{ }^{1} \mathrm{H}$ study. Magn Reson Med 2001b; 45:61-70. [PubMed: 11146487]

Duong TQ, Kim DS, Ugurbil K, Kim SG. Localized blood flow response at sub-millimeter columnar resolution. Proc Natl Acad Sci U S A 2001c;98:10904-10909. [PubMed: 11526212]

Ferris CF, Snowdon CT, King J, Duong TQ, Ziegler TE, Ugurbil K, Ludwig RL, Schultz-Darken N, Wu Z, Olson DP, Sullivan J, Tannenbaum P, Vaughan JT. Functional imaging of brain activity in conscious monkeys responding to sexually arousing cues. NeuroReport 2001;12:2231-2236. [PubMed: 11447340]

Flecknell, PA.; Waynsorth, HB. Experimental and surgical techniques in rats. San Diego: Academic Press; 1992.

Gelb AW, Bayona NA, Wilson JX, Cechetto DF. Propofol anesthesia compared to awake reduces infarct size in rats. Anesthesiology 2002;96:1183-1190. [PubMed: 11981160]

Heiss WD, Traupe H. Comparison between hydrogen clearance and microsphere technique for rCBF measurement. Stroke 1981;12:161-167. [PubMed: 7233459]

Hendrich KS, Kochanek PM, Melick JA, Schiding JK, Statler KD, Williams DS, Marion DW, Ho C. Cerebral perfusion during anesthesia with fentanyl, isoflurane, or pentobarbital in normal rats studied by arterial spin-labeling MRI. Magn Reson Med 2001;46:202-206. [PubMed: 11443729]

Hensen TD, Warner DS, Todd MM, Vust LJ, Trawick DC. Distribution of cerebral blood flow during halothane versus isoflurane anesthesia in rats. Anesthesiology 1988;69:332-337. [PubMed: 3415014]

Herscovitch P, Raichle ME. What is the correct value for the brain-blood partition coefficient for water? J Cereb Blood Flow Metab 1985;5:65-69. [PubMed: 3871783]

Hoffman WE, Edelman G, Kochs E, Werner C, Segil L, Albrecht RF. Cerebral autoregulation in awake versus isoflurane-anesthetized rats. Anesth Analg 1991;73:753-757. [PubMed: 1952176]

Hoge RD, Atkinson J, Gill B, Crelier GR, Marrett S, Pike GB. Investigation of BOLD signal dependence on cerebral blood flow and oxygen consumption: the deoxyhemoglobin dilution model. Magn Reson Med 1999;42:849-863. [PubMed: 10542343]

$\mathrm{Hu} \mathrm{X}$, Le TH, Ugurbil K. Evaluation of the early response in fMRI in individual subjects using short stimulus duration. Magn Reson Med 1997;37:877-884. [PubMed: 9178239]

Jacewicz, M.; Pulsinelli, WA. Stroke pathophysiology, diagnosis and management. Barnett, HMJ.; Mohr, JP.; Stein, BM.; Yatsu, FM., editors. Philadelphia, PA: Churchill Livingston; 1986.

Kawaguchi M, Kimbro JR, Drummond JC, Cole DJ, Kelly PJ, Patell PM. Isoflurane delays but does not prevent cerebral infarction in rats subjected to focal ischemia. Anesthesiology 2000;92:1226-1228. [PubMed: 10781264]

Kemna LJ, Posse S. Effect of respiratory $\mathrm{CO} 2$ changes on the temporal dynamics of the hemodynamic response in functional MR imaging. NeuroImage 2001;14:642-649. [PubMed: 11506537]

Kim DS, Duong TQ. Mapping cortical columnar structures using fMRI. Physiol Behav 2002;77:641644. [PubMed: 12527012]

Kim DS, Duong TQ, Kim SG. High-resolution mapping of iso-orientation columns by fMRI. Nature Neurosci 2000;3:164-169. [PubMed: 10649572]

Kim SG. Quantification of relative cerebral blood flow change by flow-sensitive alternating inversion recovery (FAIR) technique: application to functional mapping. Magn Reson Med 1995;34:293-301. [PubMed: 7500865]

Kim SG, Ugurbil K. Comparison of blood oxygenation and cerebral blood flow effects in fMRI: estimation of relative oxygen consumption change. Magn Reson Med 1997;38:59-65. [PubMed: 9211380]

Kwong KK, Belliveau JW, Chesler DA, Goldberg IE, Weisskoff RM, Poncelet BP, Kennedy DN, Hoppel BE, Cohen MS, Turner R, Cheng HM, Brady TJ, Rosen BR. Dynamic magnetic resonance imaging of human brain activity during primary sensory stimulation. Proc Natl Acad Sci U S A 1992;89:56755679. [PubMed: 1608978] 
Lahti KM, Ferris CF, Li F, Sotak CH, King JA. Imaging brain activity in conscious animals using functional MRI. J Neurosci Methods 1998;82:75-82. [PubMed: 10223517]

Liebeskind DS, Kasner SE. Neuroprotection for ischemic stroke: an unattainable goal? CNS Drugs 2001;15:165-174. [PubMed: 11463125]

Logothetis NK, Guggenberger H, Peled S, Pauls J. Functional imaging of the monkey brain. Nature Neurosci 1999;2:555-562. [PubMed: 10448221]

Maekawa T, Tommasino C, Shapiro HM, Keifer-Goodman J, Kohlemberger RW. Local cerebral blood flow and glucose utilization during isoflurane anesthesia in rat. Anesthesiology 1986;65:144-151. [PubMed: 3740503]

Manohar M, Parks C. Regional distribution of brain and myocardial perfusion in swine while awake and during 1.0 and 1.5 MAC isoflurane anesthesia produced without or with $50 \%$ nitrous oxide. Cardiovasc Res 1984;18:344-353. [PubMed: 6744355]

Matta BF, Heath KJ, Tipping K, Summors AC. Direct cerebral vasodilatory effects of sevoflurane and isoflurane. Anesthesiology 1999;91:677-680. [PubMed: 10485778]

Menon RS, Ogawa S, Hu X, Strupp JP, Anderson P, Ugurbil K. BOLD-based functional MRI at 4 Tesla includes a capillary bed contribution: echo planar imaging correlates with previous optical imaging using intrinsic signals. Magn Reson Med 1995;33:453-459. [PubMed: 7760717]

Miyawaki T, Matsui K, Takashima S. Developmental characteristics of vessel density in the human fetal and infant brains. Early Hum Dev 1998;53:65-72. [PubMed: 10193927]

Molinari, GF. Stroke pathophysiology, diagnosis and management. Barnett, HMJ.; Mohr, JP.; Stein, BM., et al., editors. London: Churchill Livingston; 1986.

Nagase K, Iida H, Ohata H, Dohi S. Ketamine, not propofol, attenuates cerebrovascular response to carbon dioxide in humans with isoflurane anesthesia. J Clin Anesth 2001;13:551-555. [PubMed: 11755322]

Ogawa S, Tank DW, Menon R, Ellermann JM, Kim SG, Merkle H, Ugurbil K. Intrinsic signal changes accompanying sensory stimulation: functional brain mapping with magnetic resonance imaging. Proc Natl Acad Sci U S A 1992;89:5951-5955. [PubMed: 1631079]

Parkes LM, Tofts PS. Improved accuracy of human cerebral blood perfusion measurements using arterial spin labeling: accounting for capillary water permeability. Magn Reson Med 2002;48:27-41. [PubMed: 12111929]

Peeters RR, Tindemans I, Schutter ED, Van der Linden A. Comparing BOLD fMRI signal changes in the awake and anesthetized rat during electrical forepaw stimulation. Magn Reson Imaging 2001;19:821-826. [PubMed: 11551722]

Posse S, Elghahwagi B, Wiese S, Kiselev VG. Effect of graded hypo- and hypercapnia on fMRI contrast in visual cortex: quantification of $\mathrm{T}_{2} *$ changes by multiecho EPI. Magn Reson Med 2001;46:264271. [PubMed: 11477629]

Reinstrup P, Ryding E, Algotsson L, Messeter K, Asgeirsson B, Uski T. Distribution of blood flow during anesthesia with isoflurane or halothane in humans. Anesthesiology 1995;82:359-366. [PubMed: 7856894]

Sharp, P.; LaRegina, M. Animal pocket reference series. Washington, DC: CRC Press; 1998.

Silva A, Williams D, Koretsky A. Evidence for the exchange of arterial spin-labeled water with tissue water in rat brain from diffusion-sensitized measurements of perfusion. Magn Reson Med 1997a; 38:232-237. [PubMed: 9256102]

Silva AC, Zhang W, Williams DS, Koretsky AP. Estimation of water extraction fractions in rat brain using magnetic resonance measurement of perfusion with arterial spin labeling. Magn Reson Med 1997b;37:58-68. [PubMed: 8978633]

Silva A, Lee SP, Yang C, Iadecola C, Kim SG. Simultaneous BOLD and perfusion functional MRI during forepaw stimulation in rats. J Cereb Blood Flow Metab 1999;19:871-879. [PubMed: 10458594]

Sokoloff L, Reivich M, Kennedy C, Des Rosiers M, Patlak G, Pettigrew K, Sakurada O, Shinohara M. The $\left[{ }^{14} \mathrm{C}\right]$ deoxyglucose method for the measurement of local cerebral glucose utilization: theory, procedure, and normal values in the conscious and anesthetized albino rat. J Neurochem 1977;28:897-916. [PubMed: 864466]

Strebel S, Kaufmann M, Baggi M, Zenklusen U. Cerebrovascular carbon dioxide reactivity during exposure to equipotent isoflurane and isoflurane in nitrous oxide anesthesia. $\mathrm{Br} \mathrm{J}$ Anaesth 1993;71:272-276. [PubMed: 8123406] 
Strupp JP. Stimulate: a GUI based fMRI analysis software package. NeuroImage 1996;3:S607.

Stullken EHJ, Milde JH, Michenfelder JD, Tinker JH. The nonlinear responses of cerebral metabolism to low concentrations of halothane, enflurane, isoflurane, and thiopental. Anesthesiology 1977;46:28-34. [PubMed: 831591]

Tsekos NV, Zhang F, Merkle H, Nagayama M, Iadecola C, Kim SG. Quantitative measurements of cerebral blood flow in rats using the FAIR technique: correlation with previous iodoantipyrine autoradiographic studies. Magn Reson Med 1998;39:564-573. [PubMed: 9543418]

Ueki M, Miles G, Hossmann KA. Effect of alpha-chloralose, halothane, pentobarbital and nitrous oxide anesthesia on metabolic coupling in somatosensory cortex of rat. Acta Anesthesiol Scand 1992;36:318-322.

Vanduffel W, Fize D, Mandeville JB, Nelissen K, Van Hecke P, Rosen BR, Tootell RB, Orban GA. Visual motion processing investigated using contrast agent-enhanced fMRI in awake behaving monkeys. Neuron 2001;32:565-577. [PubMed: 11719199]

Warner DS, McFarlane C, Todd MM, Ludwig P, McAllister AM. Sevoflurane and halothane reduce focal ischemic brain damage in the rat: possible influence on thermoregulation. Anesthesiology 1993;79:985-992. [PubMed: 8239017]

Warner DS, Ludwig PS, Pearlstein R, Brinkous AD. Halothane reduces focal ischemic injury in the rat when brain temperature is controlled. Anesthesiology 1995;82:1237-1245. [PubMed: 7741299]

Wei HM, Weiss HR, Sinha AK, Chi OZ. Effects of nitric oxide synthase inhibition on regional cerebral blood flow and vascular resistance in conscious and isoflurane-anesthetized rats. Anesth Analg 1993;77:880-885. [PubMed: 7692769]

Weiss HR, Buchweitz E, Sinha AK. Effect of hypoxic-hypercapnia on cerebral regional oxygen consumption and supply. Circ Res 1983;51:494-503. [PubMed: 7127684]

Wong EC, Buxton RB, Frank LR. Quantitative imaging of perfusion using a single subtraction (QUIPSS and QUIPSSII). Magn Reson Med 1998;39:702-708. [PubMed: 9581600]

Wyrwicz AM, Chen N, Li L, Weiss C, Disterhoft JF. fMRI of visual system activation in the conscious rabbit. Magn Reson Med 2000;44:474-478. [PubMed: 10975901]

Yacoub E, Le T, Ugurbil K, Hu X. Further evaluation of the initial negative response in functional magnetic resonance imaging. Magn Reson Med 1999;41:436-441. [PubMed: 10204863]

Young WL, Barkai AI, Prohovnik I, Nelson H, Durkin M. Effect of $\mathrm{PaCO}_{2}$ on cerebral blood flow distribution during halothane compared with isoflurane anesthesia in the rat. $\mathrm{Br} \mathrm{J}$ Anaesth 1991;67:440-446. [PubMed: 1931402]

Zeman, W.; Innes, JRM. Craigies's neuroanatomy of the rat. New York: Academic Press; 1963.

Zhang Z, Andersen AH, Avison MJ, Gerhardt GA, Gash DM. Functional MRI of apomorphine activation of the basal ganglia in awake rhesus monkeys. Brain Res 2000;852:290-296. [PubMed: 10678755]

Zhou J, van Zijl PC. Effect of transit times on quantification of cerebral blood flow by the FAIR $\mathrm{T}_{1}$ difference approach. Magn Reson Med 1999;42:890-894. [PubMed: 10542347]

Zhou J, Wilson DA, Ulatowski JA, Traystman RJ, van Zijl PC. Two-compartment exchange model for perfusion quantification using arterial spin tagging. J Cereb Blood Flow Metab 2001;21:440-455. [PubMed: 11323530] 


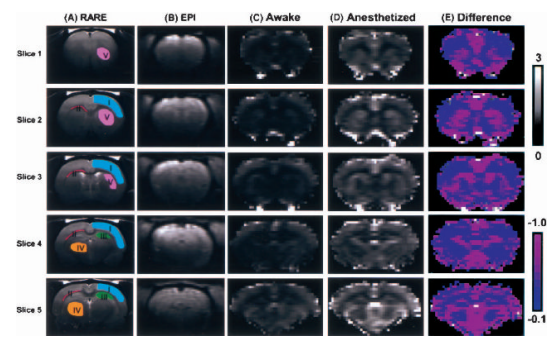

FIG. 1.

(A) Anatomic images of an awake rat obtained using fast spin-echo (rapid acquisition with relaxation enhancement [RARE] pulse sequence. Overlaid on the anatomy are representative regions of interest (ROIs) of five major structures used in quantitative cerebral blood flow (CBF) analysis, namely: I. Cerebral cortex-blue; II. corpus callosum-red; III. hippocampus - green; IV. thalamus-yellow; V. caudoputamen—pink. These ROIs, displayed on only one hemisphere for clarity, were conservatively drawn to minimize partial volume effects. (B) Representative echo-planar imaging (EPI) images of an awake rat obtained from the continuous arterial spin-labeling measurement. There are no obvious movement artifacts. Representative perfusion images of the awake (C) and anesthetized (D) conditions in the same rat. The overall cerebral blood flow (CBF) is substantially lower in the awake than in the isofluraneanesthetized rat. Cerebral blood flow under awake and anesthetized states is heterogeneously distributed. (E) The difference by subtracting anesthetized from awake CBF is also heterogeneous. Note that the two gradient-echo EPI (but not the spin-echo RARE anatomy) images show signal drop around the ear canals near the bottom of the brain. Gray scale bar indicates the $\mathrm{CBF}$ images ranging from 0 to $3 \mathrm{~mL} \cdot \mathrm{g}^{-1} \cdot \mathrm{min}^{-1}$. The color bar indicates the difference in CBF values ranging from -0.1 to $1.0 \mathrm{~mL} \cdot \mathrm{g}^{-1} \cdot \mathrm{min}^{-1}$. (F) Representative raw perfusion data obtained from the cerebral cortex and the caudoputamen over the $\approx 3$.3-minute measurement. The oscillations were $\approx 8 \%$ of the original signal (from 0.96 to 1.04), indicative of the labeled and nonlabeled images acquired in an alternate fashion. No obvious movement artifacts in the time course were observed. 


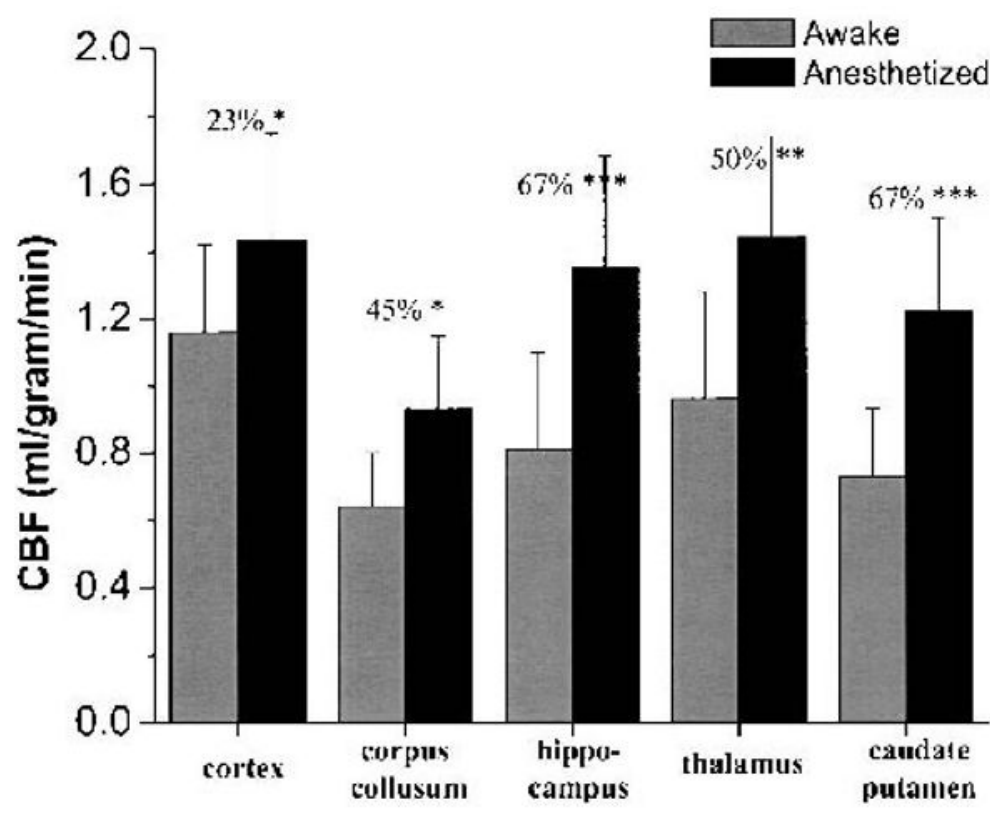

FIG. 2.

Quantitative cerebral blood flow (CBF) in awake and anesthetized rats in five major brain structures $(n=8)$. The percent differences above the bar graphs are the CBF increases by isoflurane anesthesia relative to the awake state. $* P \leq 0.005, * * P \leq 10^{-4} ; * * * P \leq 10^{-7}$. Error bars are standard deviations. 

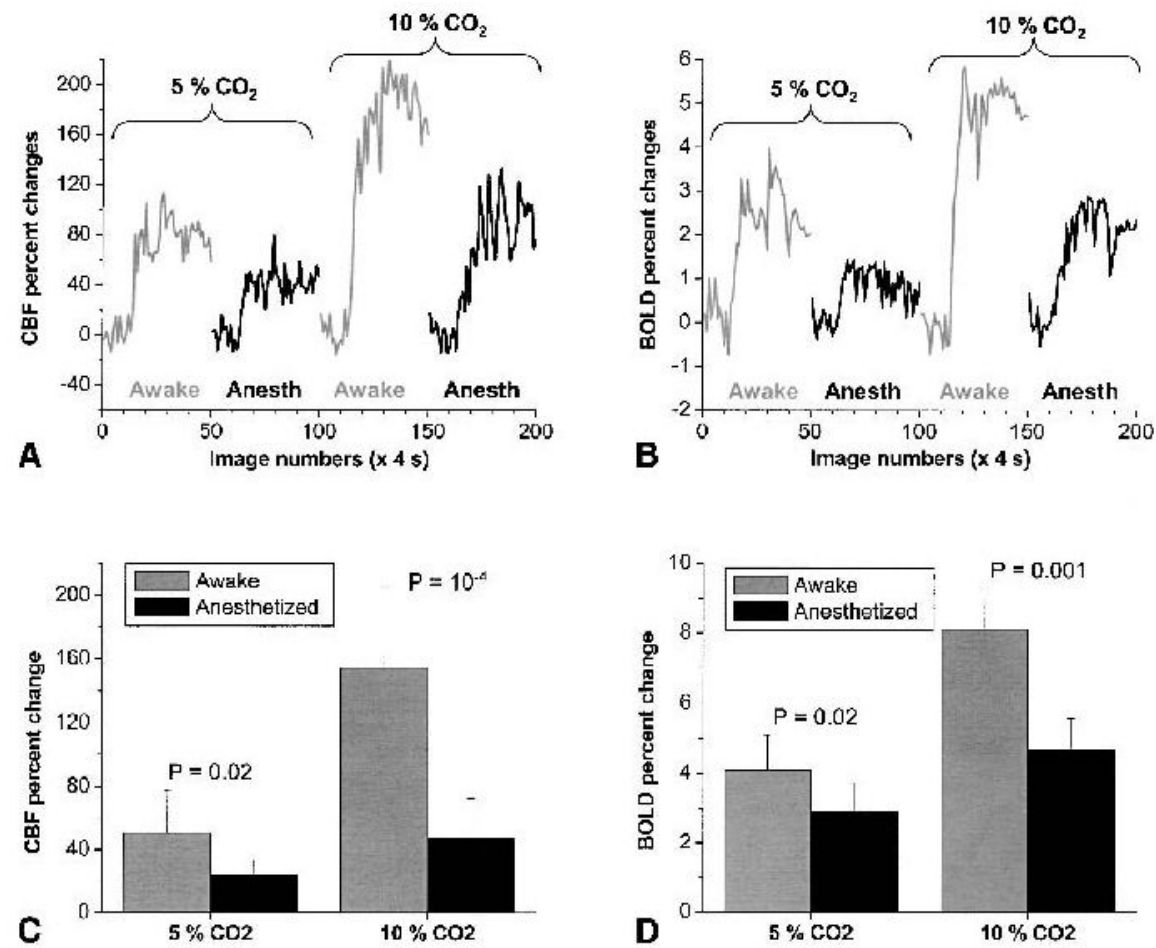

FIG. 3.

Representative cerebral blood flow (CBF) (A) and blood oxygen level-dependent (BOLD) (B) time courses from an animal without signal averaging. Four time courses, namely, 5\% $\mathrm{CO}_{2}$ under awake (gray) and anesthetized (black) conditions and $10 \% \mathrm{CO}_{2}$ under awake (gray) and anesthetized (black) conditions were concatenated. Averaged CBF (C) and BOLD (D) percent changes during hypercapnic $\left(5 \%\right.$ and $\left.10 \% \mathrm{CO}_{2}\right)$ challenge in awake and anesthetized conditions from 7 animals. Error bars are standard deviations. The $P$ values are comparison between awake and anesthetized conditions. 


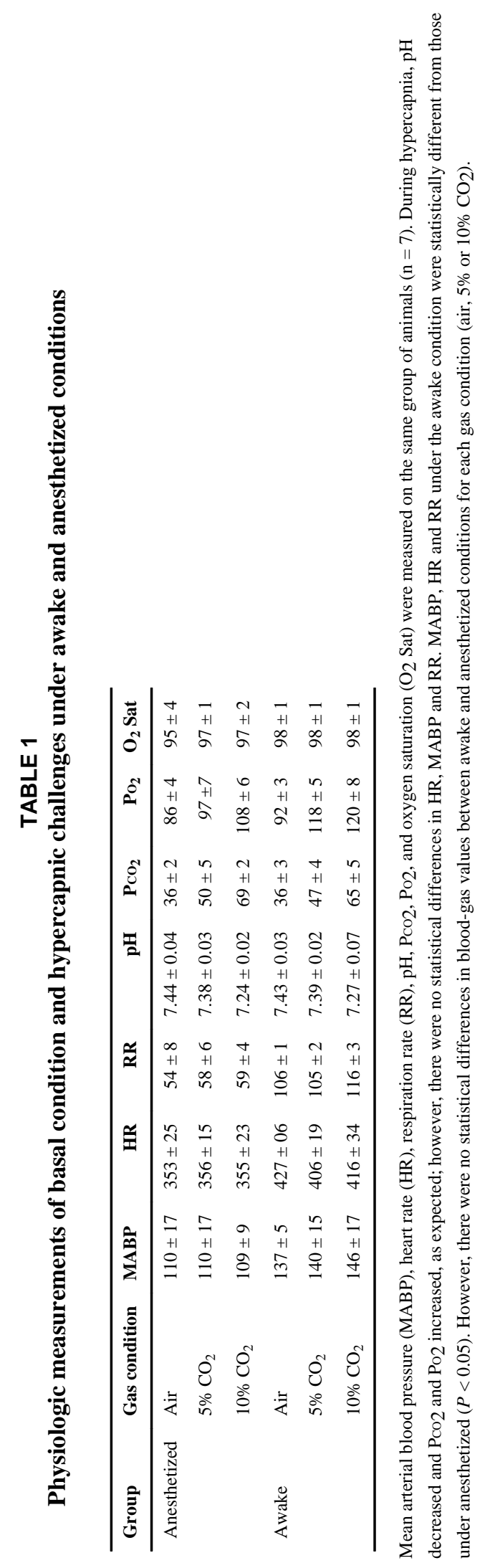

J Cereb Blood Flow Metab. Author manuscript; available in PMC 2010 November 22. 